\title{
Comparison Between Single and Two-Antenna VNA System Used for Heartbeat Rate Extraction
}

\author{
Sarah El-Samad ${ }^{1,2}$, Dany Obeid ${ }^{1}$, Gheorghe Zaharia ${ }^{2}$, Sawsan Sadek ${ }^{1}$, Ghais El Zein ${ }^{2}$ \\ ${ }^{1}$ Doctoral School of Science and Technology, Lebanese University, Lebanon \\ ${ }^{2}$ IETR - UMR CNRS 6164, INSA Rennes, France \\ Sarah.samad@insa-rennes.fr
}

\begin{abstract}
This paper presents a microwave system for measuring the cardiopulmonary activity performed in two ways: by using single antenna and by using two antennas. This system generates a continuous wave signal at $20 \mathrm{GHz}$ toward the target and then measure the reflected signal. The target is the person's chest set placed at a distance of $1 \mathrm{~m}$. The VNA measures the phase variation between the emitted and received signals. This phase variation contains information about the movement of the chest. An electrocardiograph is used simultaneously with the system as reference in order to determine the accuracy of the VNA system. The measurements are performed for a person under two conditions: breathing normally and holding the breath. Wavelet transform is used for the processing the obtained signals and the heartbeat rate is extracted accurately.
\end{abstract}

Keywords-single-antenna VNA system; two-antennas VNA system; electrocardiograph; heartbeat rate; wavelet decomposition

\section{INTRODUCTION}

Nowadays, contactless monitoring of the patient's heartbeat using Doppler radar has attracted considerable interest of researchers, especially when the electrocardiogram (ECG) measurements is not practical in some cases like infants at risk, sudden infant syndrome or burn victims. According to Doppler effect, a constant frequency signal reflected off an object having a varying displacement will result in a reflected signal, but with a time-varying phase [1]. In our case, the object is the patient's chest; the reflected signal of the person's chest contains information about the heartbeat and respiration. The radar system used is based on antennas and a Vector Network Analyzer (VNA) that measures the time-varying $S_{21}$ phase. Processing techniques are used to extract the heartbeat signal from the time-varying phase; then heartbeat rate is calculated.

Previous works have demonstrated the possibility of detecting the heartbeat activity with two-antenna VNA system, for different operating frequencies and several radiated powers [2]. In addition, a study of a single-antenna VNA system operation at $16 \mathrm{GHz}$ was realized for a person while holding his breath [3].

In this work, a comparison between single-antenna VNA system and two-antenna VNA system for heartbeat measurement is proposed for a person who is holding his breath. The system is tested at an operational frequency of 20
GHz. Measurements are carried out simultaneously with a PCbased electrocardiograph (ECG) and the relative error between system and ECG results is computed. The goal of this work is to evaluate the accuracy of the single-antenna system relatively to the widely used two-antenna system. Noteworthy to mention that using a single antenna system will reduce the number of components to be used; hence it will reduce the cost. Measurements are performed at different radiated powers: $-7,-12$, and $-17 \mathrm{dBm}$. In the case of two-antenna system, $S_{21}$ is measured, while $S_{11}$ is measured when using a single-antenna VNA system. These parameters present a timevarying phase. After detecting both parameters $S_{11}$ and $S_{21}$, processing techniques are used in order to extract the heartbeat signal, hence the heartbeat rate.

The rest of this paper is organized as follows: section II presents the measurement setup of the two systems and the obtained results when holding breath. Section III presents results of a single antenna VNA system for a breathing person. Section IV concludes the work.

\section{COMPARISON BETWEEN HEARTBEAT RATES EXTRACTED FROM SINGLE AND TWO-ANTENNA VNA SYSTEM FOR A NON-BREATHING PERSON}

\section{A. Measurement Setup}

For both designs, when using one and two antennas, the system is based on a Vector Network Analyzer. Horn antennas (LB-42-25-C2-SF) operating between $18-26.5 \mathrm{GHz}$ are used. The operating frequency is chosen to be $20 \mathrm{GHz}$. The choice of this frequency is made for two reasons: The first one is to obtain higher phase variation, as it is directly proportional to the frequency, and the second reason is due to the equipment's limitation $(2-20 \mathrm{GHz}$ of the VNA). The gain of the antenna at $20 \mathrm{GHz}$ is $24 \mathrm{~dB}$ [2]. The numbers of samples extracted for the signals measured with the VNA is 12801. The measurement duration is 23 seconds; hence the sampling frequency is $557 \mathrm{~Hz}$. This duration is chosen to avoid too long stopping breathing when the person is holding his breath. The measurements were performed for a 54-years person sitting at 1 meter from the antenna. Considering the antenna gain (24 $\mathrm{dB})$, the cables losses $(2 \mathrm{~dB})$ and the emitted power $(-29,-34$ and $-39 \mathrm{dBm})$, the radiated powers are $-7,-12$ and $-17 \mathrm{dBm}$, respectively to the emitted power. 
Measurements with several values of the emission power allow determining the minimum power required to accurately extract the heartbeat rate while limiting the risks of exposure to the waves for the patient and the medical staff. In addition, an ECG is used simultaneously with the system in order to evaluate the system accuracy. Fig. 1 presents the measurement system with single-antenna and an ECG.

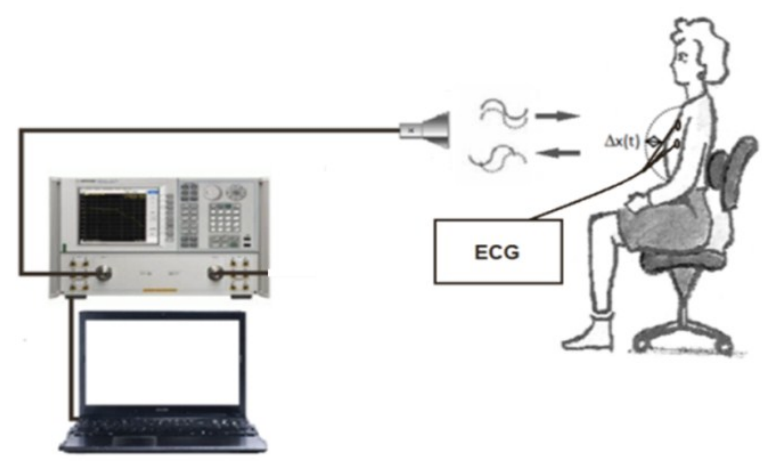

Fig. 1. Measurement system with single antenna vs. electrocardiograph.

\section{B. Signal Processing and Obtained Results}

In this section, measurements were performed for a person holding his breath when the system is used in both designs: one-antenna and two-antennas. Figs. 2 and 3 present the phase variation (PV) of $S_{11}$ and $S_{21}$ respectively, at different radiated powers: $-7,-12$ and $-17 \mathrm{dBm}$.
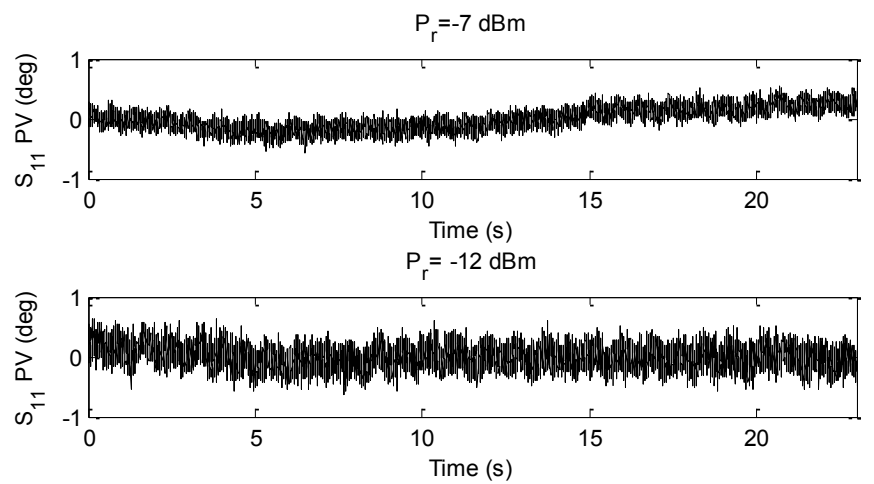

$P_{r}=-17 \mathrm{dBm}$

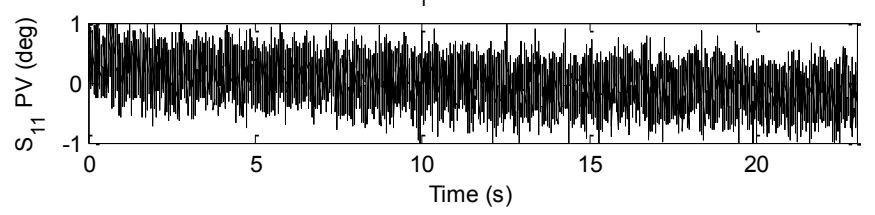

Fig. 2. Phase variation of $S_{11}$ for a person holding his breath for different radiated powers using the single-antenna VNA system.
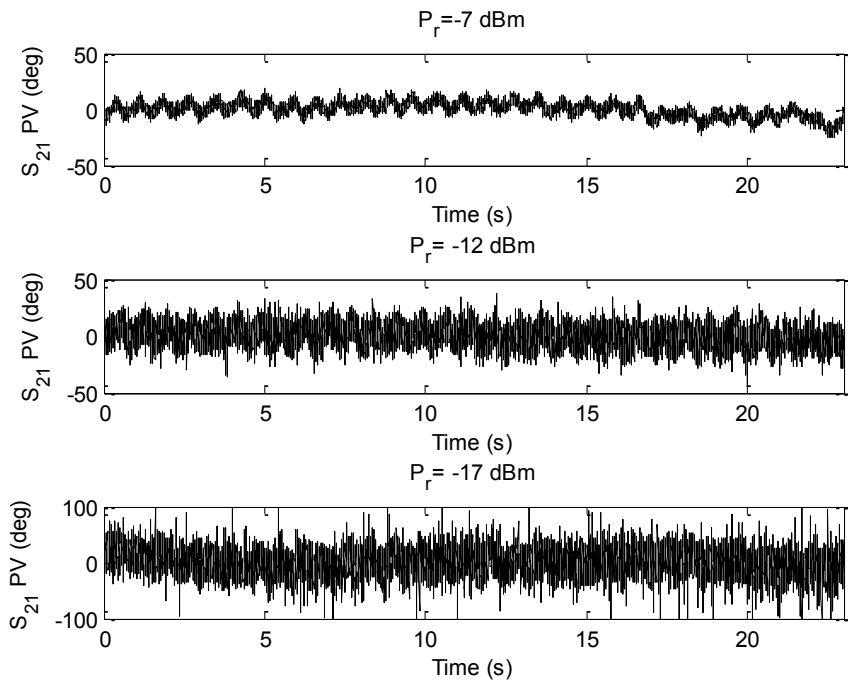

Fig. 3. Phase variation of $S_{21}$ of a person holding his breath for different radiated powers using the two-antenna VNA system.

It is remarkable that the phase variation of $S_{11}$ is more noisy and smaller than the phase variation of $S_{21}$ and the heartbeat signals are clearer in case of $S_{21}$ phase variation. The SNR of the signal decreases with the radiated power in both cases. As signals are noisy, especially at lower powers, smoothing method is used in order to reduce noise and extract the heartbeat signal by using sliding average that is applied on every $n$ consecutive samples of the waveform. After trial and errors, $n=199$ was chosen ( $n$ must be an odd number). Then, peak detection is applied in order to extract the heartbeat rate. Fig. 4 presents the peak detection of the phase variation of $S_{11}$ when using the single-antenna VNA system, after applying smoothing with $n=199$, and for different radiated powers: -7 , -12 and $-17 \mathrm{dBm}$.

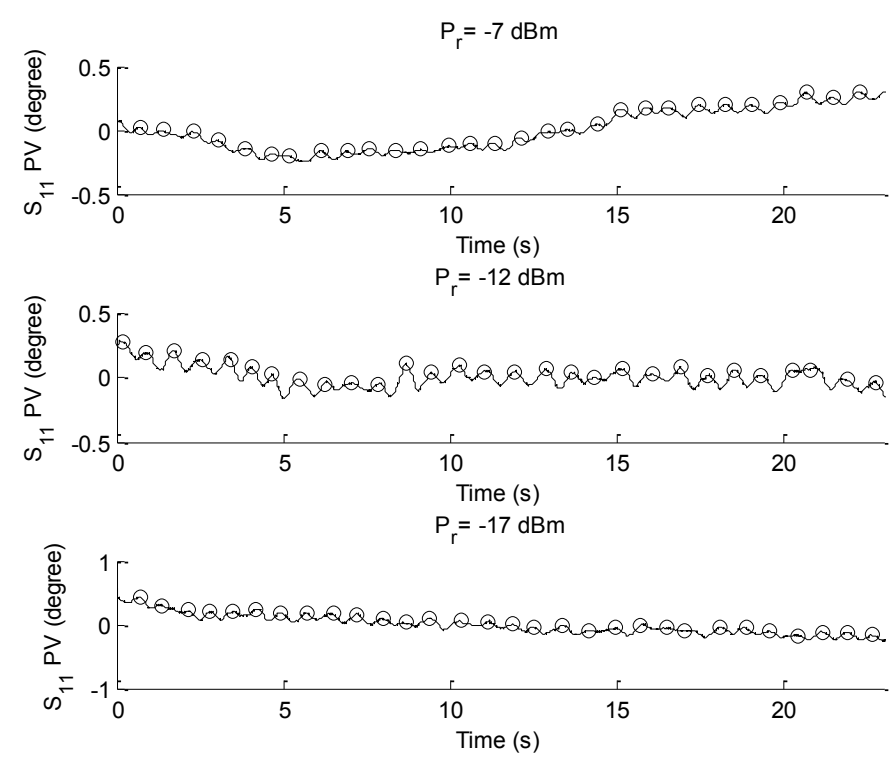

Fig. 4. Peak detection for the phase variation of $S_{11}$ after applying smoothing with $n=199$, for different radiated powers. 
After peak detection of the heartbeat signal, heartbeat rate is calculated using the following relation:

$$
H R=\frac{60(N-1)}{d_{1}+d_{2}+\cdots+d_{N-1}}
$$

where $N$ is the peaks number and $d_{k}$ is the duration of the interval determined by 2 successive peaks expressed in seconds. In case of single-antenna VNA system and at -7 $\mathrm{dBm}, 29$ peaks are obtained in $21.46 \mathrm{sec}$. At $-12 \mathrm{dBm}, 29$ peaks are obtained in $22.57 \mathrm{sec}$ and finally at $-17 \mathrm{dBm}, 30$ peaks are obtained in $21.91 \mathrm{sec}$. Hence, the heartbeat rates obtained for the person who is holding his breath are 78, 74, and $79 \mathrm{bpm}$, respectively. The same procedure is applied when using two-antennas. Recall that heartbeat rate is extracted also from the reference ECG signal taken simultaneously with VNA system. Relative error between the $H R$ of VNA system and $H R$ of ECG system is calculated using the following relation

$$
\text { RelativeError }=100 \times \frac{H R_{V N A}-H R_{E C G}}{H R_{E C G}}
$$

Table I presents the heartbeat rate extracted from the single-antenna system $\left(H R_{V N A-S A}\right)$, the heartbeat rate extracted from the ECG $\left(H R_{E C G}\right)$ and finally the relative error between $H R_{V N A}$ and $H R_{E C G}$. Table II presents the heartbeat rate extracted from the two-antenna system $\left(H R_{V N A-T A}\right)$, the heartbeat rate extracted from the ECG $\left(H R_{E C G}\right)$ and the relative error between $H R_{V N A}$ and $H R_{E C G}$.

TABLE I. Heartbeat Rate Comparison BetweEn $H R_{V N A-S A}$ AND $H R_{E C G}$

\begin{tabular}{|c|c|c|c|}
\hline $\begin{array}{c}\text { Radiated power } \\
(\mathbf{d B m})\end{array}$ & $\boldsymbol{H} \boldsymbol{R}_{V N A-S A}(\mathbf{b p m})$ & $\boldsymbol{H R}_{E C G}(\mathbf{b p m})$ & $\begin{array}{c}\text { Relative } \\
\text { Error (\%) }\end{array}$ \\
\hline-7 & 78 & 72 & 8 \\
\hline-12 & 74 & 79 & 7 \\
\hline-17 & 79 & 73 & 8 \\
\hline
\end{tabular}

TABle II. HeartBeAt RAte COMPaRison BetweEn $H R_{V N A-T A}$ AND $H R_{E C G}$

\begin{tabular}{|c|c|c|c|}
\hline $\begin{array}{c}\text { Radiated power } \\
(\mathbf{d B m})\end{array}$ & $\begin{array}{c}\boldsymbol{H} \boldsymbol{R}_{\text {VNA-TA }} \\
(\mathbf{b p m})\end{array}$ & $\boldsymbol{H} \boldsymbol{R}_{E C G} \quad \mathbf{( b p m )}$ & $\begin{array}{c}\text { Relative } \\
\text { Error (\%) }\end{array}$ \\
\hline-7 & 77 & 72 & 7 \\
\hline-12 & 81 & 76 & 7 \\
\hline-17 & 80 & 75 & 7 \\
\hline
\end{tabular}

According to the American National Standard [4], medical devices measuring the heartbeat rate should have a relative error lower than $10 \%$ or $5 \mathrm{bpm}$; hence the obtained results are acceptable for both systems with maximum error of $8 \%$.

After holding breath, the next step is performing measurements using single-antenna VNA system for a breathing person to show the possibility of extracting heartbeat using this system.

\section{RESUlts For NoRMALly BREATHING PERSON USING SINGLE-ANTENNA VNA SYSTEM}

\section{A. Measurement Setup}

Similar measurements are performed for the same person at $20 \mathrm{GHz}$ using the single-antenna system. In this case, the person was breathing normally and sitting at $1 \mathrm{~m}$ from the system with always the ECG system used as reference. Fig. 5 presents the phase variation of $S_{11}$ for a breathing person at different radiated powers: $-7,-12$ and $-17 \mathrm{dBm}$.
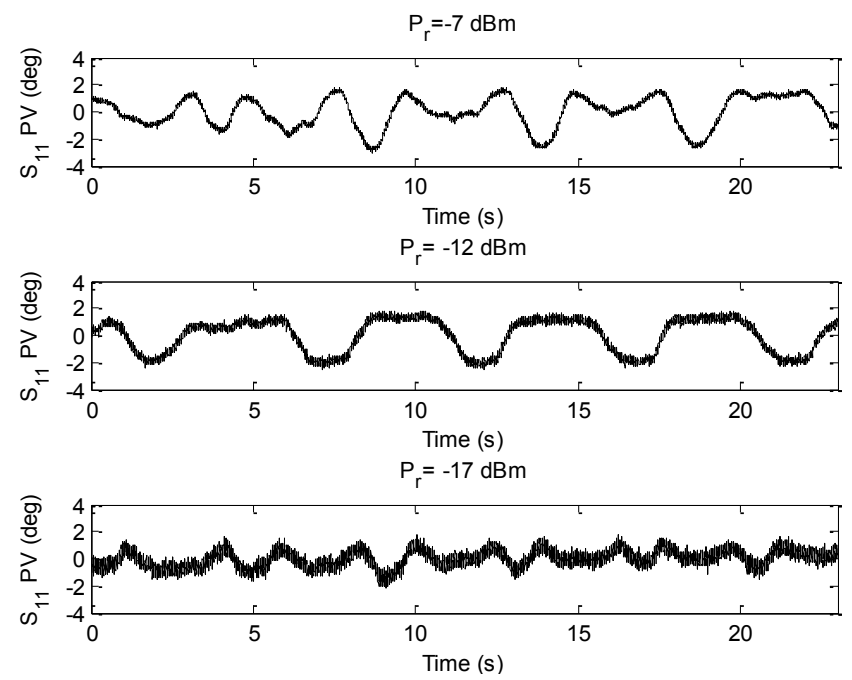

Fig. 5. Phase variation of $S_{11}$ for a breathing person for different transmitted powers.

As shown, the phase variation due to the respiration is added to the signal. In addition, the SNR decreases with the radiated power. Signal processing is required to eliminate respiration and noise in order to extract the heartbeat signal.

\section{B. Signal Processing and Obtained Results}

Wavelet decomposition is used as processing technique. The principles of wavelet transform consist of splitting the signal into many signals; each of them corresponds to an interval of frequencies. There are two types of wavelets: continuous wavelet transform (CWT) and discrete wavelet transform (DWT). DWT is interesting in the context of fast and non-redundant transforms. The DWT is the signal processing tool used in order to extract the heartbeat signal, hence, the heartbeat rate, from the cardiopulmonary signal. The DWT $\left(W_{j, k}\right)$ of a signal $f(t)$ is given by the scalar product of $f(t)$ with the scaling function (i.e. the wavelet basis function) $\varnothing(t)$ which is scaled and shifted: 


$$
W_{j, k}=<f(t), \emptyset_{j, k}(t)>=\int_{-\infty}^{+\infty} f(t) \emptyset_{j, k}(t) d t
$$

The basis function is given by:

$$
\emptyset_{j, k}(t)=2^{-j / 2} \emptyset\left(2^{-j} t-k T_{S}\right)
$$

where $j$ is the $j^{\text {th }}$ decomposition level or step and $k$ is the $k^{\text {th }}$ wavelet coefficient at the $j^{\text {th }}$ level [5]. The variables $j$ and $k$ are integers that scale and dilate $\emptyset$ to generate wavelets. Wavelets have several families like Daubechies, Coiflets, Symlets, etc. The difference between wavelet families makes compromise between how compactly they are localized in time and how smooth they are. Within each wavelet family, the number of coefficients and the iterations used in the wavelets are classified as subclasses [5]. Wavelet decomposition is applied to extract the heartbeat signal. The choice of the suitable wavelet family depends on data. DWT decomposes the signal into approximation ' $A$ ' and detail information ' $D$ '. These are computed by successive low-pass and high-pass filtering of the discrete time-domain signal where ' $A$ ' represents the low-pass filtered signal and ' $D$ ' represents the high-pass filtered signal [5]. The decomposition is repeated to increase the frequency resolution. In general, if $n$ is the decomposition number, $A_{n}$ contains frequencies between 0 and $f_{s} / 2^{n+1}$ and $D_{n}$ contains frequencies between $f_{s} / 2^{n+1}$ and $f_{s} / 2^{n}$, where $f_{s}$ is the sampling frequency and $N$ is the decomposition level $(n=1,2 \ldots N)$. The decomposed signal can be reconstructed using the following relation:

$$
S_{\text {reconstructed }}=A_{N}+D_{N}+D_{N-1}+\cdots+D_{1}
$$

As not all the wavelet families fulfill the properties of the desired signal, the chosen wavelet family has to be as close as possible to the analyzed signal to give a better reconstruction with fewer decomposition levels [6]. The choice of a suitable wavelet is based on the perfect reconstruction of the power system signal. The error between the original signal and the reconstructed signal should be the smallest for perfect reconstruction. The Root Mean Square Error (RMSE) is the indicator to measure the error between the original signal $x(n)$ and the reconstructed signal $\hat{x}(n)$. It is given by:

$$
R M S E=\sqrt{\frac{\sum_{n=0}^{N-1}|x(n)-\hat{x}(n)|^{2}}{N}}
$$

where $N$ is the samples number of the signal $x$.

RMSE between the reconstructed and original signals are calculated for all wavelet types; hence Bior 2.4 is chosen because it has the lowest RMSE compared to the other wavelets families [7]. Resampling is used to change the sampling frequency to $512 \mathrm{~Hz}$; hence $S_{\text {reconstructed }}-A_{8}$ that contains frequencies higher than $1 \mathrm{~Hz}$ is extracted. Smoothing method using sliding average with $n=199$ is applied to
$S_{\text {reconstructed }}-A_{8}$, and then peak detection is applied to the smoothed $S_{\text {reconstructed }}-A_{8}$. Table III presents $H R_{V N A-S A}$ and $H R_{E C G}$ for a breathing person with different radiated powers and the relative error between them.

TABLE III. HEARTBEAT RATE COMPARISON BetweEn $H R_{V N A-S A}$ AND $H R_{E C G}$ FOR A BREATHING PERSON WITH DIFFERENT RADIATED POWERS

\begin{tabular}{|c|c|c|c|}
\hline $\begin{array}{c}\text { Radiated power } \\
(\mathbf{d B m})\end{array}$ & $\begin{array}{c}H R_{V N A-S A} \\
\mathbf{( b p m )}\end{array}$ & $\begin{array}{c}\boldsymbol{H R}_{E C G} \\
\mathbf{( b p m )}\end{array}$ & $\begin{array}{c}\text { Relative Error } \\
\mathbf{( \% )}\end{array}$ \\
\hline-7 & 90 & 77 & 17 \\
\hline-12 & 82 & 80 & 3 \\
\hline-17 & 86 & 83 & 4 \\
\hline
\end{tabular}

Heart-rates are extracted even at low power and are less than $4 \%$; hence, the obtained results are acceptable. However, at $-7 \mathrm{dBm}$, the relative error is $17 \%$, hence new measurements should be done at $-7 \mathrm{dBm}$ to ensure the results and lead to a good interpretation.

\section{CONCLUSION}

Although signals of single-antenna VNA system are noisier than signals with two antennas, the system still able to measure heartbeat rate and its relative errors are comparable to those with two-antenna VNA system for the person who holds his breath. Concerning the breathing person, after applying wavelets, one antenna microwave system is able to extract heart rate successfully. Future work will focus on performing measurement in different scenarios like presence of random body movement, obstacles, clutters, etc.

\section{REFERENCES}

[1] J. C. Lin, J. Kiernicki, M. Kernicki, and P. B. Wollschlaeger, "Microwave Apexcardiography", IEEE Trans. on Microwave Theory and Techniques, 1979, Vol. 27, No. 6, pp. 618-620.

[2] D. Obeid, S. Sadek, G. Zaharia, G. El Zein, "Noncontact heartbeat detection at 2.4, 5.8 and $60 \mathrm{GHz}$ : A comparative study", Microwave and Optical Technology Letter, Vol. 51, No. 3, pp. 666-669, March 2009.

[3] D. Obeid, G. Zaharia, S. Sadek, G. El Zein, "ECG vs. single-antenna system for heartbeat activity detection", 4th International Symposium on Applied Sciences in Biomedical and Communication Technologies (ISABEL), October 2011, Barcelona, Spain.

[4] American National Standard, "Cardiac monitors, heart rate meters, and alarms", ANSI/AAMI EC1.

[5] G. A. Blackburn, J. Garke Ferwerda, "Retrieval of chlorophyll concentration from leaf reflectance spectra using wavelet analysis", Remote Sensing of Environment, Vol. 112, No. 4, pp. 1614-1632, April 2008.

[6] C. Stolojescu, I. Railean, S. Moga, A. Isar, "Comparison of wavelet families with application to WiMAX traffic forecasting", International Conference on Optimization of Electrical and Electronic Equipment, May 2010, Brasov, Romania.

[7] D. Obeid, S. El-Samad, G. Zaharia, S. Sadek, G. El Zein, "Advanced signal processing techniques for microwave cardiopulmonary signals separation", International Journal on Biology and Biomedical Engineering, Vol. 10, ISSN: 1998-4510, November 2016, Rome. 